\title{
Antifungal and immunomodulatory activity of Allium jesdianum Boiss extracts
}

\author{
Alireza Naeini ${ }^{\mathbb{D}}$, Roya Yaraee $^{2^{\mathbb{D}}}$, Hojjatollah Shokri ${ }^{3 *}$ \\ ${ }^{1}$ Department of Parasitology and Mycology, Faculty of Medicine and Traditional Medicine Clinical Trail Research Center, Shahed University, Tehran, \\ Iran \\ ${ }^{2}$ Department of Immunology, Medical Faculty, Shahed University, Tehran, Iran \\ ${ }^{3}$ Department of Pathobiology, Faculty of Veterinary Medicine, Amol University of Special Modern Technologies, Amol, Iran
}

\section{A R T I C L E I N F O}

Article Type:

Original Article

\section{Article History:}

Received: 26 May 2019

Accepted: 6 July 2019

\section{Keywords:}

Immunomodulatory activity

Allium jesdianum

Macrophage viability

NO production

Candida albicans

\begin{abstract}
A B S T RA C T
Introduction: Macrophages are one of the key phagocytes against various pathogenic fungi, particularly Candida species killed by various mechanisms such as nitric oxide (NO) agents. The purposes of this research were to investigate the anti-Candida and immunomodulatory effects of the extracts from Allium jesdianum on mouse peritoneal macrophages.

Methods: The antifungal assay of amphotericin B and nystatin, as well as hydroalcoholic extract from A. jesdianum was carried out using disk diffusion and broth macrodilution methods against Candida albicans (ATCC 10231). Furthermore, microculture tetrazolium (MTT) and nitrite assays (Griess test) were applied to study the influence of the aqueous extract from $A$. jesdianum on macrophage viability indices and NO production, respectively.

Results: The results showed inhibition zone values of $8,16,28 \mathrm{~mm}$ for $A$. jesdianum, amphotericin $\mathrm{B}$ and nystatin against the organism tested, respectively. The minimum inhibitory concentration (MIC) and minimum fungicidal concentration (MFC) of $A$. jesdianum were found to be 330 and $663 \mu \mathrm{g} / \mathrm{mL}$, respectively. Aqueous extract of A. jesdianum induced a significant decrease (2.8fold at concentration of $5 \mathrm{mg} / \mathrm{mL}$ and 4.3 -fold at concentration of $10 \mathrm{mg} / \mathrm{mL}$ ) in macrophage viability indices in comparison with control group $(P<0.001)$ but there was no toxic effect at 1 and $0.5 \mathrm{mg} / \mathrm{mL}$. In addition, the aqueous extract of $A$. jesdianum resulted in a significant increase in NO production at non-toxic concentrations $(77.6 \mu \mathrm{M}$ nitrite at concentration of 1 $\mathrm{mg} / \mathrm{mL}$ and $79.4 \mu \mathrm{M}$ at concentration of $0.5 \mathrm{mg} / \mathrm{mL})$ by macrophages $(P<0.01)$.

Conclusion: The extract of $A$. jesdianum showed an in vitro anti-C. albicans and NO stimulatory effect. More studies with purified immunomodulatory components of $A$. jesdianum should be performed in future to shed light on the exact mechanisms of this activity.
\end{abstract}

Implication for health policy/practice/research/medical education:

Allium jesdianum revealed good antifungal effect against $C$. albicans, so it is suggested as an alternative medicine to treat disseminated candidiasis. In addition, A. jesdianum can be used to increase macrophage functions.

Please cite this paper as: Naeini A, Yaraee R, Shokri H. Antifungal and immunomodulatory activity of Allium jesdianum Boiss extracts. J Herbmed Pharmacol. 2020;9(1):75-80. doi: 10.15171/jhp.2020.11.

\section{Introduction}

It is believed that macrophages, dendritic cells, and natural killer cells mediate the innate immune system (1). Macrophages are the key components of the mononuclear phagocytes consisting of closely related cells of bone marrow origin, involving blood monocytes and tissue macrophages. Macrophages are involved in all stages of the immune response and are capable of releasing numerous compounds like nitric oxide (NO) (2). NO has cytotoxic features, produced during host defense against invasive organisms and immunologic reactions, and is involved in numerous physiologic processes of mammals such as neurotransmition, blood pressure control and inflammation (3).

As the number of immunocompromised patients is on the rise and broad-spectrum antifungal agents, abdominal surgery, indwelling central venous lines, parenteral nutrition and cytotoxic chemotherapy are being used greatly; disseminated candidiasis has paved its way as an important cause of mortality and morbidity (4). In 
spite of recent advances in antifungal therapy, the cure rate of disseminated candidiasis is still not satisfactory due to lack of nontoxic effective antifungal agents having satisfactory pharmacokinetic features (5). It is argued that developing azoles provide new options to treat or prevent disseminated candidiasis. Yet, the development of resistance to antifungal azoles is a new challenge to the restricted therapeutic strategies (6). New agents with potent antifungal effectiveness, enhanced safety and high levels of tissue penetration are obviously required.

Many natural compounds obtained from herbal plants are believed to modify the biological response and modulate immune response $(7,8)$. It is believed that herbal extracts/essential oils boost immune response by up-regulating $\mathrm{NO}$ from peritoneal macrophages acting as effective immunomodulator and antifungal substances (9). Among the plants of the genus Allium (family Amaryllidaceae), Allium jesdianum Boiss (Bon-e-Sorkh or Lizak in Persian, Sourah Boneh in Kurdish) is one of the main species of this genus. It is an endemic Iranian plant naturally grown in northern, western, and southwestern regions of Iran. Iranians have used its medicinal and antimicrobial features from ancient times (10). Since studies on the pharmacological features of $A$. jesdianum are scarce (11), the current study aims to explore the effect of A. jesdianum extracts on growth inhibition of Candida albicans and $\mathrm{NO}$ production by applying murine peritoneal macrophages to reveal the possible immunomodulatory features.

\section{Materials and Methods \\ Plant}

The aerial sections of $A$. jesdianum were harvested from Chaharmahal and Bakhtiari province, western part of Iran, in 2018. Botanical identification was performed at the Herbarium of Pharmacognosy Department, Faculty of Medicine, Shahed University, Iran. All experiments were performed at Faculty of Medicine, Shahed University, Tehran, Iran.

Preparation of hydroalcoholic and aqueous extracts Aerial parts of the plant were crushed into fine powder. The hydroalcoholic and aqueous extracts from A. jesdianum were prepared in this study. For hydroalcoholic extract preparation, $100 \mathrm{~g}$ of the plant powder was extracted with $50 \%$ ethanol $+50 \%$ water employing maceration method (12). Whatman paper (No. 42) was used to filter the extract and the solvent was distilled at $60^{\circ} \mathrm{C}$. The remaining extract was ultimately put to dry in an oven at $30^{\circ} \mathrm{C}$ for 3 hours for removing any residual solvent, and was kept at $4^{\circ} \mathrm{C}$ until further analysis. For aqueous extract preparation, $100 \mathrm{~g}$ of the plant powder was mixed with 400 $\mathrm{mL}$ of water and boiled for $10 \mathrm{~min}$. The resulting solution was frozen and lyophilized for 96 hours at $-50^{\circ} \mathrm{C}$ and 0.04 mbar (Snijder scientific Ltd, the Netherlands).

\section{Candida albicans strain}

Sabouraud dextrose agar (Mk Co., Darmstadt, Germany) was used to culture C. albicans (ATCC 10231) at $35^{\circ} \mathrm{C}$ for 3 days, which was then harvested and kept at $4^{\circ} \mathrm{C}$ until further analysis.

\section{Antifungal assays}

We conducted a pilot study to determine the antifungal properties of different extracts from $A$. jesdianum. Based on in vitro study, only the hydroalcoholic extract from $A$. jesdianum had anti-Candida albicans effect. Therefore, the hydroalcoholic extract was selected for antifungal assays.

\section{(A) Disk diffusion method}

Test to assess the antifungal activity was performed using disk diffusion method based on the CLSI-M44-A2 standard for yeasts (13). Briefly, agar plates (90-mm diameter) containing Mueller-Hinton agar (Merck Co., Darmstadt, Germany) accompanied by glucose (2\%) and methylene blue $(0.5 \mathrm{mg})$ was used. Sterile cotton swabs dipped in yeast suspensions, adjusted to $1 \times 10^{6}$ cells/ $\mathrm{mL}$, were inoculated on the agar surface. Hydroalcoholic extract of $A$. jesdianum ( $30 \mu \mathrm{L}$ of stock solution), amphotericin B (10 $\mu \mathrm{g} /$ disk) and nystatin (100 unit/disk) disks (Master Group, London, England) were placed on the agar surfaces. Subsequently, the media were kept at $35^{\circ} \mathrm{C}$ and read at 24 hours. After the colonies grew, the zones of inhibition around the disks were measured and recorded. All experiments were performed in duplicate. The interpretation of antifungal assay of standard drugs was done based on manufacture' guideline: zone diameters of $\geq 16 \mathrm{~mm}$ of the drugs as susceptible; zone diameters of 11 to $14 \mathrm{~mm}$ as susceptible dose dependent; and zone diameters of $\leq 10 \mathrm{~mm}$ as resistant.

\section{(B) Broth macrodilution method}

Minimum inhibitory concentration (MIC) and minimum fungicidal concentration (MFC) of hydroalcoholic extract of $A$. jesdianum was determined by broth macrodilution method (14). Briefly, sterile Sabouraud dextrose broth was used to prepare stock solution of hydroalcoholic extract. Serial dilutions of stock solution of extract were prepared for a final concentration ranging from 15 to $4000 \mu \mathrm{g} / \mathrm{mL}$ at $12 \times 75 \mathrm{~mm}$ glass tubes. Also, the specific concentrations of amphotericin B and nystatin (Sigma-Aldrich Chemicals Co., St. Louis, MO, USA) ranged from 0.016 to $16 \mu \mathrm{g} / \mathrm{mL}$ and 0.25 to $128 \mu \mathrm{g} / \mathrm{mL}$, respectively. After adding $50 \mu \mathrm{L}$ of the yeast suspension $\left(2.5 \times 10^{3} \mathrm{cell} / \mathrm{mL}\right)$ to each tube, all tubes were put for incubation at $35^{\circ} \mathrm{C}$ for 48 hours. Tubes containing only the Sabouraud dextrose broth with no microorganisms were applied as controls. The lowest hydroalcoholic extract concentration inhibiting fungal growth was recognized as MIC. For determining MFC, a loopful of broth was removed from each individual tube and spot-inoculated on individual Sabouraud dextrose 
agar plates. The plates were put for incubation at $35^{\circ} \mathrm{C}$ for 48 hours, and MFC was set as the corresponding concentrations needed for killing $99.9 \%$ of the cells.

\section{Macrophages preparation}

The Animal Breeding Laboratory of the Faculty of Medicine, Shahed University, Tehran, Iran provided the study with male Balb/c mice ( 8 to 10 weeks of age, weighting 18-25 g). All animals were housed and handled according to institutionally recommended guidelines. The animals were sacrificed and peritoneal exudates cells were harvested as described by Naeini et al (9). Briefly, the macrophages were obtained by lavage using $5 \mathrm{~mL}$ of cold PBS (5 mg/mL, pH 7.2) and poured in sterile plastic tubes. Cells were pooled, re-suspended in RPMI 1640 containing 5\% FBS (GIBCO, Grand Island, NY, USA) and cultured in 96-well flat-bottom microtiter plates at a final concentration of $4 \times 10^{5}$ cells per well. After 2 hours, the debris and non-adherent cells were taken away from the wells.

In in vitro pilot study, only the aqueous extract from A. jesdianum showed the immunomodulatory effect. Therefore, the aqueous extract was selected for MTT and nitrite assays. The monolayer macrophages were incubated once more at $37^{\circ} \mathrm{C}$ for 20 hours along with various concentrations of the aqueous extract $(0.5,1,5$ and $10 \mathrm{mg} / \mathrm{mL}$ ).

\section{MTT assay}

The effect of the aqueous extract from A. jesdianum on macrophage viability was measured by MTT [3(4, 5-dimethylthiazol-2-yl)-2, 5-diphenyltetrazolium bromide] assay (15). Macrophages were incubated with the Allium aqueous extract at different concentrations (10, 5,1 and $0.5 \mathrm{mg} / \mathrm{mL}$ ) and media. The 96-well plates were used to carry out the MTT assay. The complete medium was used to wash the wells followed by the addition of $180-\mu \mathrm{L}$ aliquots of medium and $20-\mu \mathrm{L}$ aliquots of MTT solution $(5 \mathrm{mg} / \mathrm{mL}$ of PBS) to each well at the pre-defined time. Incubation lasted for 2 hours at $37^{\circ} \mathrm{C}$ and $5 \% \mathrm{CO}_{2}$ for exponentially growing cells and 15 minutes for steadystate confluent cells; then, the media were taken away followed by solubilizing the formazan crystals with $175 \mu \mathrm{L}$ of DMSO. A microplate reader (Model 450) was used to read the plates on (Bio-Rad Laboratories, Hercules, CA, USA) at 540-nm wavelength.

\section{NO production}

With the method specified by Pertile et al, NO production was determined (16). NO discharged into the supernatants of mouse macrophages was specified through Griess reaction by addition of $50 \mu \mathrm{L}$ of the aqueous extract from A. jesdianum to 96-well flat-bottomed plates containing $50 \mu \mathrm{L}$ of Griess reagent [1\% sulfanilamide/0.1\% N-(1naphthyl) ethylenediamine dihydrochloride $\left./ 2.5 \% \mathrm{H}_{3} \mathrm{PO}_{4}\right]$.
The samples were assayed in quadruplicate. After 15 minutes at room temperature, a Multiskan MS microplate reader (Labsystems Oy, Helsinki, Finland) was used for measuring the absorbance of each well at $540 \mathrm{~nm}$ and the nitrite concentration was determined from a standard curve of sodium nitrite.

Statistical analysis

One-way analysis of variance (ANOVA) was used for data analysis and the results were shown as Mean + Standard deviation (SD). The $P$ values $<0.05$ were considered as significant differences.

\section{Results}

In disk diffusion method, the diameters of growth inhibition zones for A. jesdianum hydroalcoholic extract, amphotericin B and nystatin were 8, 16 and $28 \mathrm{~mm}$, respectively. In broth macrodilution method, C. albicans isolate was sensitive to the hydroalcoholic extract from $A$. jesdianum and this activity was found to be with MIC of $330 \mu \mathrm{g} / \mathrm{mL}$ (Table 1).

As shown in Table 2, macrophage viability indices reduced after the treatment of peritoneal macrophages at all concentrations of the aqueous extract from $A$. jesdianum, especially at 5 and $10 \mathrm{mg} / \mathrm{mL}$ concentrations. Aqueous extract from $A$. jesdianum resulted in a significant decrease of $280 \%$ at concentration of $5 \mathrm{mg} / \mathrm{mL}$ and $430 \%$ at concentration of $10 \mathrm{mg} / \mathrm{mL}$ in macrophage viability indices in comparison with control group $(P<0.001)$. Significant difference was not observed between the activity of the aqueous extract from $A$. jesdianum and control group at 1 and $0.5 \mathrm{mg} / \mathrm{mL}$ concentrations.

Regarding the effect of the aqueous extract of $A$. jesdianum on NO production, a progressive effect was observed (Figure 1). As shown in Table 2, the aqueous extract from $A$. jesdianum at the concentrations of 1 and $0.5 \mathrm{mg} / \mathrm{mL}$ stimulated a considerable increase in $\mathrm{NO}$ production in comparison with control group $(P<0.01$ and $P<0.001$, respectively). The concentrations of $\mathrm{NO}$ produced by macrophages treated with aqueous extract were $77.6 \mu \mathrm{M}$ at $1 \mathrm{mg} / \mathrm{mL}$ and $79.4 \mu \mathrm{M}$ at $0.5 \mathrm{mg} / \mathrm{mL}$. The best effect of the aqueous extract from $A$. jesdianum on NO production was observed at lower concentrations. In

Table 1. Antifungal susceptibility of Allium jesdianum hydroalcoholic extract, amphotericin B and nystatin against Candida albicans

\begin{tabular}{lccc}
\hline \multirow{2}{*}{ Groups } & \multicolumn{3}{c}{ Method } \\
\cline { 2 - 4 } & \multicolumn{2}{c}{ Disk Diffusion } & Broth macrodilution \\
\cline { 2 - 4 } & Inhibition zone $(\mathrm{mm})$ & $\begin{array}{c}\text { MIC }(\mu \mathrm{g} / \\
\mathrm{mL})\end{array}$ & $\begin{array}{c}\text { MFC }(\mu \mathrm{g} / \\
\mathrm{mL})\end{array}$ \\
\hline Allium jesdianum & 8 & 330 & 663 \\
Amphotericin B & 16 & 2 & 4 \\
Nystatin & 28 & 4 & 8 \\
\hline
\end{tabular}

MIC: Minimum inhibitory concentration; MFC: Minimum fungicidal concentration. 
Table 2. The cell viability and nitric oxide (NO) production of murine peritoneal macrophages treated with the aqueous extract from Allium jesdianum

\begin{tabular}{|c|c|c|c|c|c|c|c|}
\hline \multirow{2}{*}{ Groups } & \multirow{2}{*}{ Dose $(\mathrm{mg} / \mathrm{mL})$} & \multicolumn{3}{|c|}{ MTT assay } & \multicolumn{3}{|c|}{ NO assay $(\mu \mathrm{M})$} \\
\hline & & Mean & SD & $P$ value & Mean & SD & $P$ value \\
\hline \multirow[t]{4}{*}{ Treatment groups } & 10 & 0.083 & 0.001 & $0.001^{a}$ & 42.0 & 6.4 & - \\
\hline & 5 & 0.129 & 0.039 & $0.001^{\mathrm{a}}$ & 37.2 & 4.4 & - \\
\hline & 1 & 0.213 & 0.024 & - & 77.6 & 13.2 & $0.01^{\mathrm{a}}$ \\
\hline & 0.5 & 0.198 & 0.016 & - & 79.4 & 15.0 & $0.001^{\mathrm{a}}$ \\
\hline Control & - & 0.359 & 0.048 & - & 48.8 & 6.6 & - \\
\hline
\end{tabular}

MTT, Microculture tetrazolium; SD, standard deviation.

a Significant differences were observed between treatment and control groups.

addition, no significant differences were observed between the aqueous extract from $A$. jesdianum and control group at 5 and $10 \mathrm{mg} / \mathrm{mL}$ concentrations.

\section{Discussion}

In this study, we investigated the influence of hydroalcoholic extract from $A$. jesdianum on growth inhibition of $C$. albicans, as well as the effect of the aqueous extract from $A$. jesdianum on viability and NO production of macrophages. The present study showed inhibitory effect of hydroalcoholic extract from $A$. jesdianum against $C$. albicans. The hydroalcoholic extract showed an $8 \mathrm{~mm} / 30 \mu \mathrm{L}$ inhibition zone against the tested strain, while the growth inhibition zones of amphotericin $\mathrm{B}$ and nystatin were 16 and $28 \mathrm{~mm}$, respectively. In broth macrodilution method, the hydroalcoholic extract from A. jesdianum also exhibited an antifungal activity against tested strain with MIC value of $330 \mu \mathrm{g} / \mathrm{mL}$. Our antifungal assays demonstrated the fungistatic and fungicidal effects of hydroalcoholic extract from $A$. jesdianum against $C$. albicans. Earlier studies have reported the antifungal activity of Allium species hydroalcoholic extract (17-19). Our findings are consistent with similar studies assessing antifungal activity of Allium species hydroalcoholic extract on different fungal species (20). Shahrokh et al (11) indicated the antifungal influence of hydroalcoholic extract from $A$. jesdianum against all fluconazole resistant and susceptible Candida isolates. The $\mathrm{MIC}_{90}$ of hydroalcoholic extract from A. jesdianum against Candida strains from patients with candidiasis was $3 \mathrm{mg} / \mathrm{mL}$. In a study by Razzaghi et al (21), the MIC values of hydroalcoholic extract of $A$. jesdianum against Trichophyton mentagrophytes isolates were between 6.1 and $49.3 \mathrm{mg} / \mathrm{mL}$ (mean MIC: $20.3 \mathrm{mg} / \mathrm{mL}$ ), whereas the MFC values of the isolates were between 24.6 and 49.3 $\mathrm{mg} / \mathrm{mL}$ (mean MFC: $40.6 \mathrm{mg} / \mathrm{mL}$ ). In another study by Moghim et al (22), the $\mathrm{MIC}_{90}$ of ethanolic extract of Allium ascalonicum against C. albicans was $8.65 \mathrm{mg} / \mathrm{mL}$. Diba and Alizadeh (20) revealed that hydroalcoholic extract of Allium hirtifolium strongly inhibited the activity of Candida tropicalis isolates at a concentration of 100 $\mathrm{mg} / \mathrm{mL}$. Other studies reported that the MFC values of various extracts from Allium species against Aspergillus niger, A. flavus and A. fumigatus were between 35-1536 $\mu \mathrm{g} / \mathrm{mL}$ (23). The chemical composition and bioactive compounds of Allium plants have been reported to be organosulfur compounds, which include diallyl trisulfide, diallyl-dithiosulfinate (allicin), diallyl disulfide and S-allylcysteine $(24,25)$. Additionally, Allium plants contain polyphenolic compounds like flavonoids (26). Most of the medicinal effects of Allium genus are related to their organosulfur compounds (27). Organosulfur compounds downregulate the expression of hypha specific gene $H W P 1$, which possibly explain the inhibitory effect of

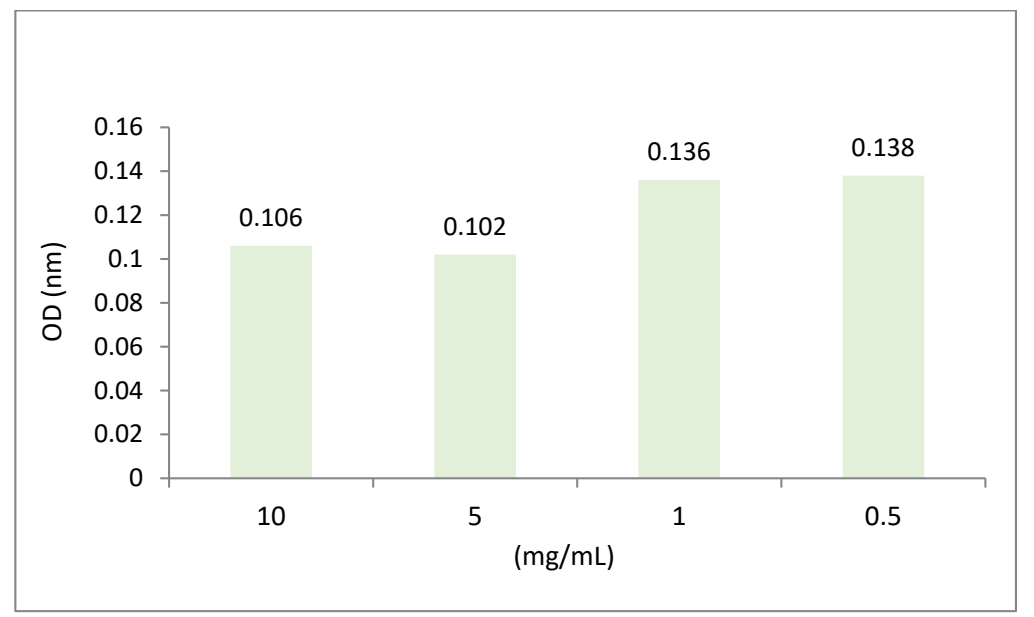

Figure 1. Nitric oxide (NO) production of peritoneal macrophages stimulated with the aqueous extract of Allium jesdianum. OD: optical density. 
Allium hydroalcoholic extract on hyphae and biofilm formation in C. albicans (5).

Phagocytic cells, particularly macrophages, are believed to be involved significantly in resistance to Candida infections. They are active in the nonspecific first line of defense due to their capability of engulfing and degrading the invading Candida yeasts (28). In the current study, we explored the influence of the aqueous extract from A. jesdianum on different aspects of macrophages. Our results showed that macrophage viability indices reduced after the treatment of peritoneal macrophages at all concentrations of the aqueous extract from A. jesdianum, especially at 5 and $10 \mathrm{mg} / \mathrm{mL}$ concentrations. Aqueous extract from $A$. jesdianum resulted in a significant decrease of 2.8 -fold at concentration of $5 \mathrm{mg} / \mathrm{mL}$ and 4.3 -fold at concentration of $10 \mathrm{mg} / \mathrm{mL}$ in macrophage viability indices in comparison with control group $(P$ $<0.001$ ). The literature review revealed a large body of research on the immunomodulatory properties of Allium species. However, there are not reports on A. jesdianum. In a study conducted by Radjabian et al (29), the tested Allium species had stimulatory or inhibitory effects on macrophages at different concentrations. Allium sativum and Allium iranicum aqueous extracts demonstrated the highest effects on macrophage viability indices at 1 and $0.01 \mathrm{mg} / \mathrm{mL}$, respectively, whereas the aqueous extract of Allium elburzense, at high concentrations, slightly affected viability indices of the macrophage. In agreement with our results, Allium asarense decreased macrophage viability indices at most applied concentrations (29). The available evidence on Allium species suggests that not only the applied aqueous extract concentration or the assessed compound, but also the types of treated cell line are effective factors on the experiment outcomes. In a previous study by Naeini et al (30), the aqueous extract of Ziziphora tenuior at the concentrations of 10 and $20 \mathrm{mg} /$ $\mathrm{mL}$ stimulated a considerable increase $(24 \%$ and $21 \%$, in the respective order) in macrophage viability as well.

The present study demonstrated that the aqueous extract from $A$. jesdianum, in a dose-dependent manner, on NO production had a progressive effect. Aqueous extract from $A$. jesdianum at the concentrations of 1 and $0.5 \mathrm{mg} / \mathrm{mL}$ stimulated a considerable increase in $\mathrm{NO}$ production in comparison with control group $(P<0.01$ and $P<0.001$, respectively). The concentrations of $\mathrm{NO}$ produced by macrophages treated with aqueous extract from $A$. jesdianum were $77.6 \mu \mathrm{M}$ at $1 \mathrm{mg} / \mathrm{mL}$ and 79.4 $\mu \mathrm{M}$ at $0.5 \mathrm{mg} / \mathrm{mL}$. The best effect of the aqueous extract from $A$. jesdianum on NO production was observed at lower concentrations. This activity could be due to the presence of flavonoids in Allium aqueous extract, which can augment the macrophage responses (31). To the best of our knowledge, no study has focused on influence of the aqueous extract from $A$. jesdianum in NO production; however, similar works have been done with various herbs.
Previous studies demonstrated that the aqueous extracts from Ziziphora tenuior (30) and Heracleum persicum (9) had significant immunostimulatory activity in NO production by macrophages. In agreement with previous studies, our research demonstrated stimulatory activity of the aqueous extract from $A$. jesdianum in production of NO by peritoneal macrophages implying that these natural substances are activators of macrophages. The presence of NO produced by macrophages in suitable concentration during infections results in immunomodulatory functions of host defense. Therefore, the induction of NO may contribute in the immunoprevention of fungal infections, especially disseminated candidiasis (32).

\section{Conclusion}

Allium jesdianum hydroalcoholic extract exhibited an anti-C. albicans activity; thus, so it is suggested as an alternative medicine to treat disseminated candidiasis. In addition, $A$. jesdianum aqueous extract can be used to increase macrophage functions. Unfortunately, the role of various components of $A$. jesdianum aqueous extract on the macrophage function is still unknown. Therefore, more studies should be done on the influence of the components on immune cells.

\section{Authors' contribution}

AN, RY and HS conceived and designed experiments and analyzed the data; HS wrote the paper. All authors read and confirmed publication of the paper.

\section{Conflict of interests}

The authors declare no conflict of interest.

\section{Ethical considerations}

The protocol of this study was confirmed by the Ethical Committee of Amol University of Special Modern Technologies, Amol, Iran (ir.ausmt.rec.1398.04.17). Ethical issues (including plagiarism, data fabrication and double publication) have been completely observed by the authors.

\section{Funding/Support}

This study was funded by Research Council of Faculty of Veterinary Medicine, Amol University of Special Modern Technologies, Amol, Iran (Grant No. 98/20/7811). Also, we are grateful to Mr. H. Smaeil-Zade Nami and Mr. D. Jamali for their kind helps.

\section{References}

1. Singh N, Tailang M, Mehta S. A review on herbal plants as immunomodulators. Int J Pharm Sci Res. 2016;7(9):360210 .

2. Hirayama D, Iida T, Nakase H. The phagocytic function of macrophage-enforcing innate immunity and tissue homeostasis. Int J Mol Sci. 2017;19(1). doi: 10.3390/ ijms 19010092 . 
3. Lok HC, Sahni S, Jansson PJ, Kovacevic Z, Hawkins CL, Richardson DR. A nitric oxide storage and transport system that protects activated macrophages from endogenous nitric oxide cytotoxicity. J Biol Chem. 2016;291(53):27042-61. doi: 10.1074/jbc.M116.763714.

4. Khosravi AR, Shokri H, EshghiS. In vitrolymphoproliferative response and cytokine production in mice with experimental disseminated candidiasis. Iran J Basic Med Sci. 2017;20(2):193-8. doi: 10.22038/ijbms.2017.8248.

5. Khodavandi A, Alizadeh F, Harmal NS, Sidik SM, Othman F, Sekawi Z, et al. Comparison between efficacy of allicin and fluconazole against Candida albicans in vitro and in a systemic candidiasis mouse model. FEMS Microbiol Lett. 2011;315(2):87-93. doi: 10.1111/j.1574-6968.2010.02170.x.

6. Sharifzadeh A, Shokri H. Antifungal activity of essential oils from Iranian plants against fluconazole-resistant and fluconazole-susceptible Candida albicans. Avicenna J Phytomed. 2016;6(2):215-22.

7. Clement F, Pramod SN, Venkatesh YP. Identity of the immunomodulatory proteins from garlic (Allium sativum) with the major garlic lectins or agglutinins. Int Immunopharmacol. 2010;10(3):316-24. doi: 10.1016/j. intimp.2009.12.002.

8. Wen CC, Chen HM, Yang NS. Developing phytocompounds from medicinal plants as immunomodulators. In: Shyur LF, Lau ASY, eds. Advances in Botanical Research. Vol 62. Academic Press; 2012. p. 197-272. doi: 10.1016/B978-0-12394591-4.00004-0.

9. Naeini AR, Shokri H, Khosravi AR. Immunostimulatory effects of aqueous extract of Heracleum persicum Desf. on mouse peritoneal macrophages. Jundishapur J Microbiol. 2013;6(4):e5373. doi: 10.5812/jjm.5373.

10. Oroji Salmasi K, Javadi H, Miri SM. Evaluation of cytogenetic diversity of different populations from five Allium species. 6th Congress of Agriculture and Sustainable Natural Resources; Tehran, Iran; 2017.

11. Shahrokh S, Vahedi G, Khosravi AR, Mahzounieh M, Ebrahimi A, Sharifzadeh A, et al. In vitro antifungal activity of aqueous-ethanolic extract of Allium jesdianum against fluconazole-susceptible and -resistant human vaginal Candida glabrata isolates. J Herbmed Pharmacol. 2017;6(3):136-40.

12. Salehi Surmaghi H. Medicinal plants and phytotherapy. Tehran: Donyaye Taghazie; 2006. p. 55-8.

13. Clinical and Laboratory Standards Institute (CLSI). Method for antifungal disk diffusion susceptibility testing of yeasts; approved guideline. 2nd ed. Wayne, PA: CLSI; 2009.

14. National Committee for Clinical Laboratory Standards (NCCLS). Reference method for broth dilution antifungal susceptibility testing of yeasts: approved standard. 2nd ed. Villanova, PA: CLSI; 2002.

15. Rao YK, Fang SH, Tzeng YM. Inhibitory effects of the flavonoids isolated from Waltheria indica on the production of NO, TNF-alpha and IL-12 in activated macrophages. Biol Pharm Bull. 2005;28(5):912-5. doi: 10.1248/bpb.28.912.

16. Pertile TL, Sharma JM, Walser MM. Reovirus infection in chickens primes splenic adherent macrophages to produce nitric oxide in response to $\mathrm{T}$ cell-produced factors. Cell Immunol. 1995;164(2):207-16. doi: 10.1006/ cimm.1995.1163.

17. Sharififar F, Pournourmohammadi S, Arabnejad M, Rastegarianzadeh R, Ranjbaran O, Purhemmaty A.
Immunomodulatory activity of aqueous extract of Heracleum persicum Desf. in mice. Iran J Pharm Res. 2010;8(4):287-92. doi: 10.22037/ijpr.2010.824.

18. Block E. Garlic and other Alliums: the lore and the science. Royal Society of Chemistry; 2010.

19. Khazaei V, Nazeri S, Piri KH, Nazeri H, Zamani N. Comparative evaluation of antibacterial properties of some medicinal plants in Iran. Asian J Med Pharm Res. 2011;1(1):6-8.

20. Diba A, Alizadeh F. In vitro and in vivo antifungal activity of Allium hirtifolium and Allium sativum. Avicenna J Phytomed. 2018;8(5):465-74.

21. Razzaghi Khezerlo S, Sharifzadeh A, Soltani M, Shokri $\mathrm{H}$, Khosravi A. Determination of genetic diversity and susceptibility of Trichophyton mentagrophytes isolates against antifungal effects of ethanolic extract of Allium jesdianum. Sci Res Iran Vet J. 2017;13(3):56-66.

22. Moghim H, Taghipoor S, Shahinfard N, Kheiri S, Heydari Z, Rafieian S. Antifungal effects of Allium ascalonicum, Marticaria chamomilla and Stachys lavandulifolia extracts on Candida albicans. J Herbmed Pharmacol. 2014;3(1):9-14.

23. Yin MC, Tsao SM. Inhibitory effect of seven Allium plants upon three Aspergillus species. Int J Food Microbiol. 1999;49(1-2):49-56. doi: 10.1016/s0168-1605(99)00061-6.

24. Amiri H. Chemical composition and antibacterial activity of the essential oil of Allium jesdianum Boiss. \& Buhse from Iran. J Med Plant. 2007;6(1):39-44.

25. Mnayer D, Fabiano-Tixier AS, Petitcolas E, Hamieh T, Nehme N, Ferrant C, et al. Chemical composition, antibacterial and antioxidant activities of six essentials oils from the Alliaceae family. Molecules. 2014;19(12):20034-53. doi: $10.3390 /$ molecules 191220034 .

26. Sadri A, Khodavandi A, Alizadeh F. Quorum-sensing quenching compounds Allium sativum, Allium hirtifolium and Allium cepa: the probable quorum- sensing quenching compounds against Candida albicans. Biosci Biotech Res Asia. 2016;13(3):1457-68. doi: 10.13005/bbra/2289.

27. Sultan MT, Butt MS, Qayyum MM, Suleria HA. Immunity: plants as effective mediators. Crit Rev Food Sci Nutr. 2014;54(10):1298-308. doi: 10.1080/10408398.2011.633249.

28. Khosravi AR. Immune responses and fungal diseases. 1st ed. Tehran: Tehran University Press; 2012. [Persian].

29. Radjabian T, Hosseinpur Yektaei Z, Ghazanfari T, Nasiri Z, Fotovvat M. The immunoregulatory effects of four Allium species on macrophages and lymphocytes viability. Immunoregulation. 2018;1(3):143-52. doi: 10.32598/ Immunoregulation.1.3.143.

30. Naeini AR, Khosravi AR, Tadjbakhsh H, Ghazanfari T, Yaraee R, Shokri H. Evaluation of the immunostimulatory activity of Ziziphora tenuior extracts. Comp Clin Path. 2010;19(5):459-63. doi: 10.1007/s00580-009-0885-9.

31. Mikaili P, Maadirad S, Moloudizargari M, Aghajanshakeri S, Sarahroodi S. Therapeutic uses and pharmacological properties of garlic, shallot, and their biologically active compounds. Iran J Basic Med Sci. 2013;16(10):1031-48.

32. Carlos IZ, Carli CBA, Maia DCG, Benzatti FP, Lopes FCM, Roese FM, et al. Immunostimulatory effects of the phenolic compounds from lichens on nitric oxide and hydrogen peroxide production. Rev Bras Farmacogn. 2009;19(4):84752. doi: 10.1590/S0102-695X2009000600009. 